\title{
Partial Trade-Credit Policy of Retailer with Exponentially Deteriorating Items
}

\author{
Biswajit Sarkar · Sharmila Saren
}

Published online: 25 November 2014

(C) Springer India Pvt. Ltd. 2014

\begin{abstract}
This model considers the strategy that supplier offers retailer a full trade-credit policy whereas retailer offers their customers a partial trade-credit policy. For such assumption, retailer can earn more profits. In this supplier-retailer model, deterioration of products is assumed as exponential. The main purpose is to minimize the retailer's annual total cost with finite replenishment rate. Graphical representations are given under different circumstances. The analytical derivation of the model is given to obtain the minimum cost. Some numerical examples for different cases are provided to illustrate the model.
\end{abstract}

Keywords Inventory · Trade-credit policy · Variable deterioration

\section{Introduction}

In the competitive market, the supplier offers a fixed time period for the retailer to pay the amount of the purchased item. This fixed time period, which is offered by the supplier to the retailer, is called the trade-credit period. In addition, in order to increase their relative market shares, the retailer offers trade-credit to the customer. For this result, the retailer can delay some payments upto the last moment of permissible period which is considered by the supplier. It is assumed that the customer must pay for products at the time of receiving from the retailer. In that case, the retailer can obtain more benefits as the retailer sells products and earns some interests from the customer. If the payment for the purchased item is given by

Dr. Sarkar is in leave on lien from Vidyasagar University.

B. Sarkar $(\bowtie)$

Department of Industrial \& Management Engineering, Hanyang University,

Ansan, Gyeonggi-do 426 791, South Korea

e-mail: bsbiswajitsarkar@gmail.com

S. Saren

Department of Applied Mathematics with Oceanology and Computer Programming,

Vidyasagar University, Midnapore 721 102, India 
the customer to the supplier within the trade credit period, then no interest is charged to the customer. On the other hand, interest is charged if the amount is not settled within the fixed time period provided by the supplier.

In this direction, Arcelus et al. [1] deduced retailer's pricing credit and inventory policies for deteriorating items in response to temporary price/credit incentives. Balkhi [2] deduced an optimal economic ordering policy with deteriorating items under different supplier tradecredits for infinite horizon case. Chang et al. [3] investigated an EOQ (economic order quantity) model for deteriorating items, in which the supplier provides a permissible delay to the purchaser if the order quantity is greater than or equal to a predetermined quantity. They characterized the optimal solution and provide an easy-to-use algorithm to obtain the optimal order quantity and replenishment time. Chen et al. [4] derived an economic order quantity model under conditionally permissible delay-in-payments. Chung [5] surveyed the inventory system as a cost minimization problem to determine the retailer's optimal inventory policy under retailer's partial trade-credit policy. Mahata [6] obtained an EPQ (economic production quantity) model to determine retailers replenishment decisions for deteriorating items under two-levels of trade-credit policy. Ouyang et al. [7] addressed a study on an inventory model for non-instantaneous deteriorating items with permissible delay-in-payments. Ouyang et al. [8] deduced an economic order quantity model by providing the facts: (1) some interest rates charged by the supplier which are not necessarily higher than the interest rate earned by the wholesaler and (2) the permissible delay period offered by the supplier is independent of the permissible delay period offered by the wholesaler. Sarkar et al. [9] extended former research models including inspection policy during production to detect defective items. Shah et al. [10] discovered an inventory system for price-sensitive and time dependent demand with twolevel trade-credit policy. Soni and Patel [11] formulated an integrated inventory model for variable production rate and price-sensitive demand rate under two-level trade-credit policy.

In an economic production quantity (EPQ) model, the basic assumption is that every produced item is perfect and the production rate occurs periodically. Lead time does not vary and there are no quantity discounts. Taft [12] first considered an EPQ (economic production quantity) model in the inventory literature. Chang [13] developed an EPQ model with consolidating shipments of imperfect quality items. Cheng [14] formulated an EPQ model for consolidating shipments of defective items. Chiu et al. [15] determined replenishment lot size and shipment policy for an extended EPQ model with delivery and quality assurance issues. Goyal and Cárdenas-Barrón [16] designed an EPQ model for defective items. Hall [17] produced the range of characteristic inventory curves in an integrated production system. He described how the system attributes define the inventory curve and the resulting EOQ/EPQ equation. Pal et al. [18] presented a mathematical model on EPQ for stochastic demand in an imperfect production system. Szendrovits and Goyal [19] formulated an EPQ model for packaging two items jointly manufactured through several stages. Cárdenas-Barrón [20] discussed an EPQ model by assuming the production of imperfect quality items. Teng et al. [21] derived a deterministic EPQ model with time-varying demand. Wee et al. [22] obtained a finite horizon EPQ problem with backorders.

Maintenance of inventories for deteriorating items is a major problem in the supply chain in any business systems. Most of the physical goods undergo decay over time. Deterioration considered in most of the above EPQ (economic production quantity) models is constant, while in most of the practical cases deterioration rate increases with time. In real life situations, it can be seen that items like fruits and vegetables, whose deterioration rate increases with time. Hence, it is better to take deterioration as variable instead of considering as constant. Further, Chakrabarty et al. [23] developed an EOQ (economic order quantity) model for items with Weibull distributed deterioration, shortages, and trended demand. Chu and Chen [24] 
suggested some inventory replenishment policies for deteriorating items in an exponentially declining market. Chung and Wee [25] formulated a short life-cycle deteriorating product in an inventory model.

Chung et al. [26] formulated two-level trade-credit policy by considering deteriorating items with continuous deterioration and expiration date. Chung and Cárdenas-Barrón [27] investigated the optimal solution of an inventory model with deteriorating items under stockdependent demand and two-level trade-credit policy. Covert and Philip [28] surveyed an EOQ model for Weibull distribution deteriorating items. Goyal and Giri [29] developed an inventory model with time varying demand, production, and deterioration rates. Hariga [30] derived an EOQ model for deteriorating items with time-varying demand. Mukhopadhyay et al. [31] discussed a joint pricing and ordering policy for deteriorating items. Sana [32] deduced an optimal selling price and lot size with time varying deterioration and partial backlogging. Sarkar [33] developed a production-inventory model by assuming probabilistic deterioration of products. Sarkar [34] discussed an EOQ model for delay-in-payments and considered the time-varying deterioration rate. Sarkar et al. [35] derived an inventory model with deteriorating items considering that component cost and selling price increase with continuous rate per unit time. Later, Sarkar and Sarkar [36], Sarkar and Sarkar [37] extended previous research works by assuming time-varying deterioration. Sarkar and Sarkar [38] deduced an EMQ (economic manufacturing quantity) model by assuming probabilistic deterioration. Sett et al. [39] provided a two-warehouse inventory model with increasing demand and time varying deterioration. Skouri et al. [40] developed inventory models with ramp type demand rate, partial backlogging, and weibull deterioration rate.

Wu et al. [41] derived an EOQ model for the retailer where: (a) the supplier provides an up-stream trade-credit and the retailer also offers a down-stream trade-credit, (b) the retailers down-stream trade-credit to the buyer not only increases sales and revenue but also opportunity cost and default risk, and (c) deteriorating items not only deteriorate continuously but also have their expiration dates. Sarkar et al. [42] extended the model of Mahata [6] with variable time-varying deterioration for the fixed lifetime products. They also included supplier's full trade-credit policy to retailer and retailers partial trade-credit policy to their customers. Further, De and Sana [43] developed an inventory model with shortages as well as demand of end customers is dependent on promotional effort and selling price simultaneously. The order quantity and shortage quantities are functionally related to promotional effort, unit selling price and time alone. See Table 1 for contribution of several authors.

This paper highlights the strategy that supplier offers retailer full trade-credit period but retailer offers their customer partial trade-credit period. Deterioration of products is considered as exponential in this model. Retailer's optimal cost function is minimized by obtaining the cycle length. The mathematical model is derived in "Mathematical Model" section. Numerical examples and sensitivity analysis are given for seven cases in "Numerical Examples" section. Finally, this paper ends with concluding remarks in "Conclusions" section.

\section{Mathematical Model}

The following notation are used to formulate the model

$\begin{array}{ll}A & \text { Ordering cost (\$/order) } \\ P & \text { Production or replenishment rate (unit/year) } \\ D & \text { Demand rate (unit/year) } \\ c & \text { Purchasing cost (\$/unit) }\end{array}$


Table 1 Contribution of the several authors

\begin{tabular}{lllll}
\hline Author(s) & EPQ & $\begin{array}{l}\text { Two-level trade- } \\
\text { credit policy }\end{array}$ & $\begin{array}{l}\text { Constant } \\
\text { deterioration }\end{array}$ & $\begin{array}{l}\text { Variable } \\
\text { deterioration }\end{array}$ \\
\hline
\end{tabular}

Arcelus et al. [1]

Balkhi [2]

Chang et al. [3]

Chen et al. [4]

Chung [5]

Mahata [6]

Ouyang et al. [7]

Ouyang et al. [8]

Sarkar et al. [9]

Shah et al. [10]

Soni and Patel [11]

Taft [12]

Chang [13]

Cheng [14]

Chiu et al. [15]

Goyal and Cárdenas-Barrón [16]

Hall [17]

Pal et al. [18]

Szendrovits and Goyal [19]

Cárdenas-Barrón [20]

Teng et al. [21]

Wee et al. [22]

Chakrabarty et al. [23]

Chu and Chen [24]

Chung and Wee [25]

Chung et al. [26]

Chung and Cárdenas-Barrón [27]

Covert and Philip [28]

Goyal and Giri [29]

Hariga [30]

Mukhopadhyay et al. [31]

Sana [32]

Sarkar [33]

Sarkar [34]

Sarkar et al. [35]

$$
\begin{aligned}
& \sqrt{ } \\
& \sqrt{ } \\
& \sqrt{ }
\end{aligned}
$$

$\checkmark$

$\sqrt{ }$

$\sqrt{ }$

$\sqrt{ }$

$\checkmark$

$\checkmark$

$\checkmark$

Sarkar and Sarkar [36]

Sarkar and Sarkar [37]

Sarkar and Sarkar [38]

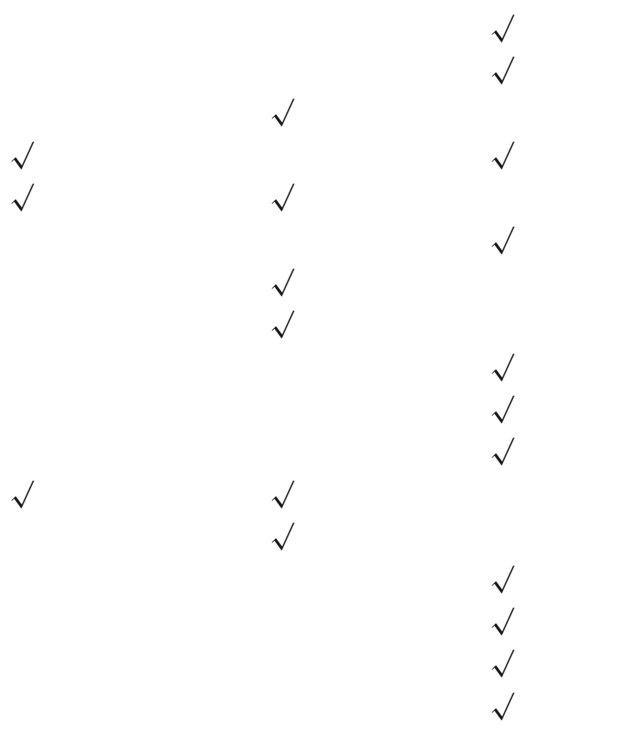

Sett et al. [39] 
Table 1 continued

\begin{tabular}{llll}
\hline Skouri et al. [40] & & & $\sqrt{ }$ \\
Wu et al. [41] & $\sqrt{ }$ & $\sqrt{ }$ \\
Sarkar et al. [42] & & & $\sqrt{ }$ \\
This paper & $\sqrt{ }$ & $\sqrt{ }$ & $\sqrt{ }$ \\
\hline
\end{tabular}

$h$

$\alpha$

M

$N$

$I_{e}$

$I_{c}$

$\theta(t)$

$t_{1}$

$T$

$\operatorname{TRC}(T)$

$T^{*}$
Selling price, $s \geq c$, (\$/unit)

Holding cost without interest charges (\$/unit/year)

Customer's fraction of the total amount owed, payable to the retailer (\$/unit)

Retailer's trade-credit period provided by the supplier (years)

Customer's trade-credit period provided by the retailer (years)

Interest earned from the customer by the supplier (\$/year)

Interest charged to the customer by the supplier (\$/year)

Time varying deterioration rate, where $0<\theta(t)<1$

Production time length in a cycle (years)

Cycle length (years)

Annual total cost (\$/year)

Optimal cycle time (years)

The following assumptions are considered to develop this model

1. The model considers constant production rate and demand rate.

2. The supplier provides full trade-credit policy to the retailer but the retailer provides partial trade-credit policy to the customer.

3. The customer can make a partial payment to the retailer when the items are sold. Thus, the retailer can earn interest from the customer's payment with rate $I_{e}$ as the customer must pay the remaining payment at the end of the trade-credit period provided by the retailer.

4. In case $T \geq M$, the account is settled at $T=M$. The retailer starts paying interest charges on items in stock with rate $I_{c}$.

5. In case $T \leq M$, the account is settled at $T=M$. The retailer does not need to pay any interest on the stock.

6. Exponential deterioration rate is considered.

7. Infinite time horizon is considered.

8. Lead time is considered as negligible.

9. Shortages are not allowed.

The model considers a production-inventory system where at time $t=0$, the production begins and raises upto the time $t=t_{1}$. Throughout the time interval $\left[0, t_{1}\right]$ the inventory level is affected by production, demand, and deterioration. At $t=t_{1}$, the production stops and after that the inventory level depletes to $t=T$ for deterioration and absorption. The graphical representation of the inventory system is given in Fig. 1.

The governing differential equation of the inventory system is

$$
\frac{d I_{1}(t)}{d t}+e^{-\theta t} I_{1}(t)=P-D, \quad 0 \leq t \leq t_{1}
$$

with the initial condition $I_{1}(0)=0$.

In the time interval $\left[t_{1}, T\right]$, there is no production, the inventory is affected by demand and deterioration. 


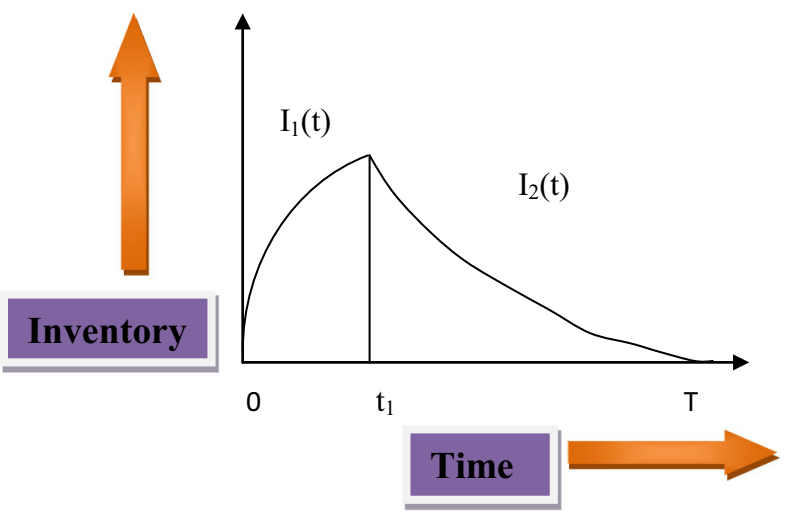

Fig. 1 Graphical representation of the inventory model

Hence the differential equation of the inventory system within $\left[0, t_{1}\right]$ is

$$
\frac{d I_{2}(t)}{d t}+e^{-\theta t} I_{2}(t)=-D, \quad t_{1} \leq t \leq T
$$

with the boundary condition $I_{2}(T)=0$.

(1) and (2) imply

$$
I_{1}(t)=(P-D)\left(t+\frac{t^{2}}{2}\right) e^{-\left(t-\frac{\theta t^{2}}{2}\right)} .
$$

and

$$
I_{2}(t)=D\left((T-t)+\frac{\left(T^{2}-t^{2}\right)}{2}\right) e^{-\left(t-\frac{\theta t^{2}}{2}\right)} .
$$

[Here the Taylor series expansion of the exponential function is taken upto second order as $\theta$ is very small.]

At $t_{1}$, from the continuity condition $I_{1}\left(t_{1}\right)=I_{2}\left(t_{1}\right)$ i.e.,

$$
t_{1}=\sqrt{1+\frac{2 D}{P}\left(T+\frac{T^{2}}{2}\right)}-1 .
$$

The annual total cost for two conditions $M \geq N$ and $M<N$.

Annual Total Cost for $M \geq N$

In this section, the annual total cost for $M \geq N$ is determined. The annual total cost is the sum of ordering cost, holding cost, deterioration cost, interest payable, and interest earned.

Annual ordering cost is $=\frac{A}{T}$.

Annual stock holding cost without interest charges is

$$
\begin{aligned}
& =\frac{h}{T}\left[\int_{0}^{t_{1}} I_{1}(t) d t+\int_{t_{1}}^{T} I_{2}(t) d t\right] \\
& =\frac{h(P-D) t_{1}^{2}}{2 T}+\frac{h D}{T}\left(\frac{T^{2}}{2}-T t_{1}+\frac{t_{1}^{2}}{2}+\frac{T t_{1}^{2}}{2}-\frac{T^{2} t_{1}}{2}+\frac{T^{2} t_{1}^{2}}{4}\right) .
\end{aligned}
$$




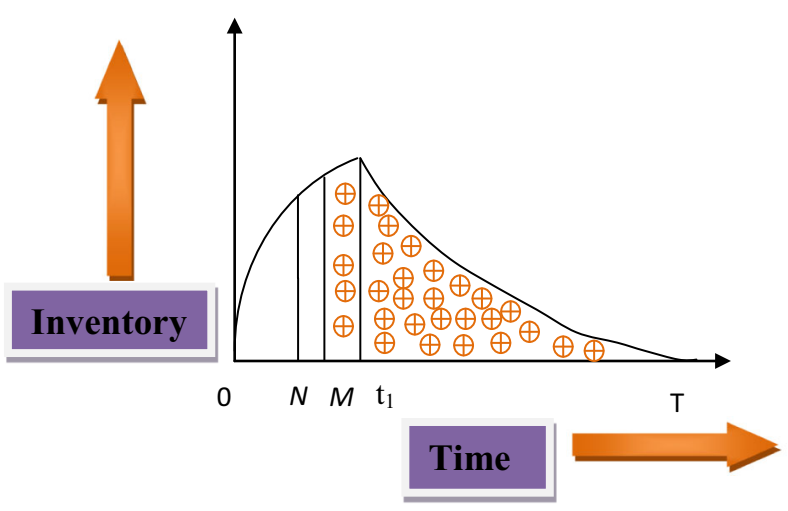

Fig. 2 Total interest payable while $T \geq t_{M}$

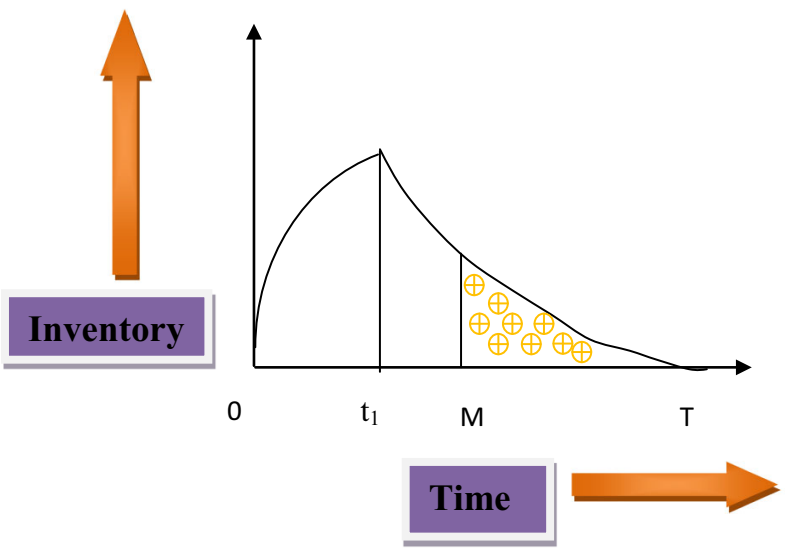

Fig. 3 Total interest payable while $M \leq T \leq t_{M}$ and $t_{1} \leq N \leq M$

Deterioration cost is $=\frac{c\left(P t_{1}-D T\right)}{T}$.

For interest charged, there are four cases as follows:

Case (i) $M \leq t_{1}$ i.e., $M \leq t_{M} \leq T$

See Fig. 2 for the inventory position.

Annual interest payable is

$$
\begin{aligned}
& =\frac{c I_{c}}{T}\left[\int_{M}^{t_{1}} I_{1}(t) d t+\int_{t_{1}}^{T} I_{2}(t) d t\right] \\
& =\frac{c I_{c}(P-D)\left(t_{1}{ }^{2}-M^{2}\right)}{2 T}+\frac{c I_{c} D}{T}\left(\frac{T^{2}}{2}-T t_{1}+\frac{t_{1}{ }^{2}}{2}+\frac{T t_{1}{ }^{2}}{2}-\frac{T^{2} t_{1}}{2}+\frac{T^{2} t_{1}{ }^{2}}{4}\right) .
\end{aligned}
$$

Case (ii) $t_{1} \leq M \leq T$ i.e., $M \leq T \leq t_{M}$

See Fig. 3 for the present inventory position.

Annual interest payable is

$$
=\frac{c I_{c}}{T}\left[\int_{M}^{T} I_{2}(t) d t\right]
$$




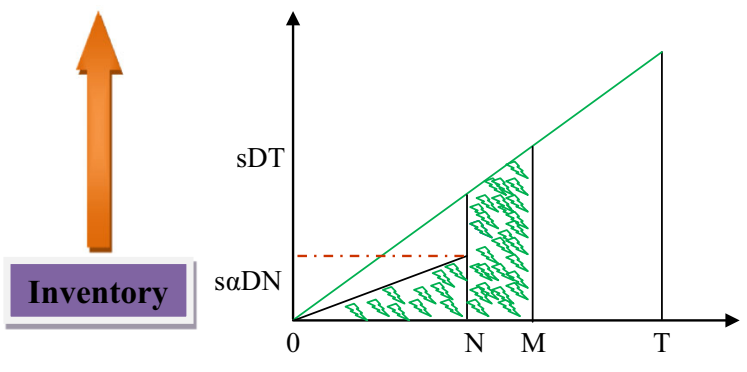

\section{Time}

Fig. 4 Total interest earned while $M \leq T$

$$
=\frac{c I_{c} D}{T}\left(\frac{T^{2}}{2}-T M+\frac{M^{2}}{2}+\frac{T M^{2}}{2}-\frac{T^{2} M}{2}+\frac{T^{2} M^{2}}{4}\right) .
$$

Case (iii) $N \leq T \leq M$

For this interval,

The annual interest payable is 0 .

Case (iv) $0<T \leq N$

The annual interest payable is 0 .

There are four cases for interest earned per year.

Case (a) $M \leq t_{1}$ i.e., $M \leq t_{M} \leq T$

See Fig. 4 for the present inventory position.

Annual interest earned is

$$
\begin{aligned}
& =\frac{s I_{e}}{T}\left[\frac{D N^{2} \alpha}{2}+\frac{(D N+D M)(M-N)}{2}\right] \\
& =\frac{s I_{e} D}{2 T}\left[M^{2}-(1-\alpha) N^{2}\right] .
\end{aligned}
$$

Case (b) $t_{1} \leq M \leq T$ i.e., $M \leq T \leq t_{M}$

Annual interest earned is $=\frac{s I_{e} D}{2 T}\left[M^{2}-(1-\alpha) N^{2}\right]$.

Which is same as Case (a)

Case (c) $N \leq T \leq M$

See Fig. 5 for the present inventory position.

Annual interest earned is

$$
\begin{aligned}
& =\frac{s I_{e}}{T}\left[\frac{D N^{2} \alpha}{2}+\frac{(D T+D N)(T-N)}{2}+(M-T) D T\right] \\
& =\frac{s I_{e} D}{2 T}\left[2 M T-(1-\alpha) N^{2}-T^{2}\right] .
\end{aligned}
$$

Case (d) $0<T \leq N$

See Fig. 6 for the present inventory position.

Annual interest earned is

$$
\begin{aligned}
& =\frac{s I_{e}}{T}\left[\frac{D T^{2} \alpha}{2}+\alpha D T(N-T)+(M-N) D T\right] \\
& =s I_{e} D\left[M-(1-\alpha) N-\frac{\alpha T}{2}\right] .
\end{aligned}
$$




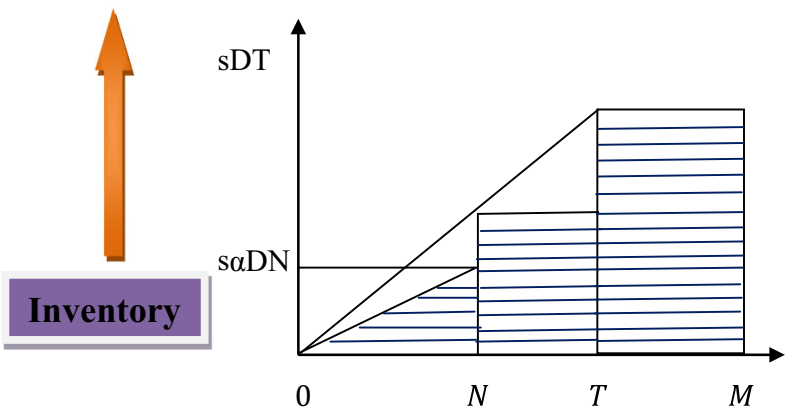

\section{Time}

Fig. 5 Total interest earned while $N \leq T \leq M$

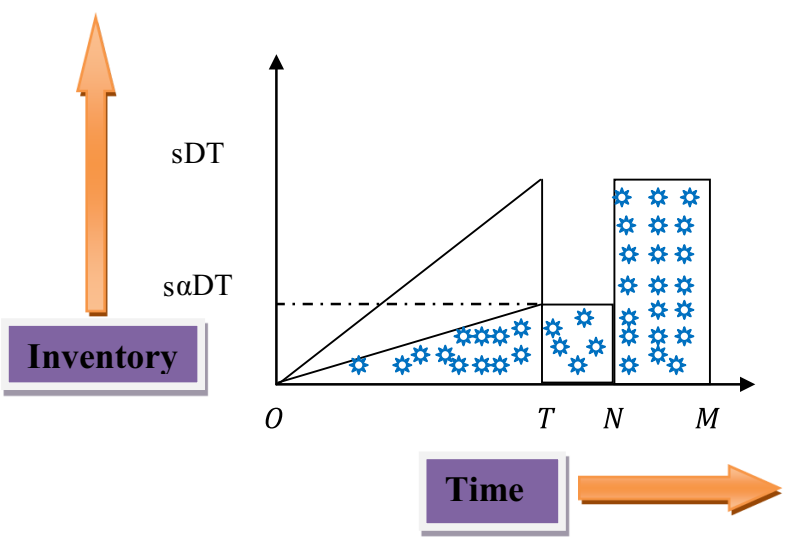

Fig. 6 Total interest earned while $0<T \leq N$

The retailer's annual total cost is given by

$$
\begin{aligned}
\operatorname{TRC}(T)= & \text { Ordering cost }+ \text { Holding cost }+ \text { Deterioration cost }+ \text { Interest payable } \\
& - \text { Interest earned. } \\
\operatorname{TRC}(T)= & \left\{\begin{array}{ll}
T R C_{1}(T), & \text { if } T \geq t_{M} \\
T R C_{2}(T), & \text { if } M \leq T \leq t_{M} \\
T R C_{3}(T), & \text { if } N \leq T \leq M \\
T R C_{4}(T), & \text { if } 0<T \leq N
\end{array}\right\}
\end{aligned}
$$

where the cost expressions are given by

$$
\begin{aligned}
T R C_{1}(T)= & \frac{A}{T}+\frac{h(P-D) t_{1}{ }^{2}}{2 T}+\frac{h D}{T}\left(\frac{T^{2}}{2}-T t_{1}+\frac{t_{1}{ }^{2}}{2}+\frac{T t_{1}{ }^{2}}{2}-\frac{T^{2} t_{1}}{2}+\frac{T^{2} t_{1}{ }^{2}}{4}\right) \\
& +\frac{c I_{c}(P-D)\left(t_{1}{ }^{2}-M^{2}\right)}{2 T}+\frac{c I_{c} D}{T}\left(\frac{T^{2}}{2}-T t_{1}+\frac{t_{1}{ }^{2}}{2}+\frac{T t_{1}{ }^{2}}{2}-\frac{T^{2} t_{1}}{2}\right. \\
& \left.+\frac{T^{2} t_{1}{ }^{2}}{4}\right)+\frac{c\left(P t_{1}-D T\right)}{T}-\frac{s I_{e} D}{2 T}\left[M^{2}-(1-\alpha) N^{2}\right],
\end{aligned}
$$




$$
\begin{aligned}
T R C_{2}(T)= & \frac{A}{T}+\frac{h(P-D) t_{1}{ }^{2}}{2 T}+\frac{h D}{T}\left(\frac{T^{2}}{2}-T t_{1}+\frac{t_{1}{ }^{2}}{2}+\frac{T t_{1}{ }^{2}}{2}-\frac{T^{2} t_{1}}{2}+\frac{T^{2} t_{1}{ }^{2}}{4}\right) \\
& +\frac{c I_{c} D}{T}\left(\frac{T^{2}}{2}-T M+\frac{M^{2}}{2}+\frac{T M^{2}}{2}-\frac{T^{2} M}{2}+\frac{T^{2} M^{2}}{4}\right)+\frac{c\left(P t_{1}-D T\right)}{T} \\
& -\frac{s I_{e} D}{2 T}\left[M^{2}-(1-\alpha) N^{2}\right], \\
T R C_{3}(T)= & \frac{A}{T}+\frac{c\left(P t_{1}-D T\right)}{T}-\frac{s I_{e} D}{2 T}\left[2 M T-(1-\alpha) N^{2}-T^{2}\right]+\frac{h(P-D) t_{1}{ }^{2}}{2 T} \\
& +\frac{h D}{T}\left(\frac{T^{2}}{2}-T t_{1}+\frac{t_{1}^{2}}{2}+\frac{T t_{1}{ }^{2}}{2}-\frac{T^{2} t_{1}}{2}+\frac{T^{2} t_{1}{ }^{2}}{4}\right),
\end{aligned}
$$

and

$$
\begin{aligned}
T R C_{4}(T)= & \frac{A}{T}+\frac{h(P-D) t_{1}{ }^{2}}{2 T}+\frac{h D}{T}\left(\frac{T^{2}}{2}-T t_{1}+\frac{t_{1}^{2}}{2}+\frac{T t_{1}^{2}}{2}-\frac{T^{2} t_{1}}{2}+\frac{T^{2} t_{1}^{2}}{4}\right) \\
& +\frac{c\left(P t_{1}-D T\right)}{T}-s I_{e} D\left[M-(1-\alpha) N-\frac{\alpha T}{2}\right] .
\end{aligned}
$$

As, $t_{M}=\sqrt{1+\frac{2 P}{D}\left(M+\frac{M^{2}}{2}\right)}-1$ and from the continuity condition at $t_{M}$, Thus, $T R C_{1}\left(t_{M}\right)=T R C_{2}\left(t_{M}\right), T R C_{2}\left(t_{M}\right)=T R C_{3}\left(t_{M}\right), T R C_{3}\left(t_{M}\right)=T R C_{4}\left(t_{M}\right), T R C(T)$, $T R C_{1}(T), T R C_{2}(T), T R C_{3}(T)$, and $T R C_{4}(T)$ are well defined for $T>0$.

Annual Total Cost for $M<N$

In this case, the annual total cost for $M<N$ is obtained. The annual total cost consists of the ordering cost, holding cost, deterioration cost, interest payable, and the interest earned during the period of time.

Annual ordering cost is $=\frac{A}{T}$.

Annual stock holding cost without interest charges is

$$
\begin{aligned}
& =\frac{h}{T}\left[\int_{0}^{t_{1}} I_{1}(t) d t+\int_{t_{1}}^{T} I_{2}(t) d t\right] \\
& =\frac{h(P-D) t_{1}^{2}}{2 T}+\frac{h D}{T}\left(\frac{T^{2}}{2}-T t_{1}+\frac{t_{1}^{2}}{2}+\frac{T t_{1}^{2}}{2}-\frac{T^{2} t_{1}}{2}+\frac{T^{2} t_{1}^{2}}{4}\right) .
\end{aligned}
$$

Deterioration cost is $=\frac{c\left(P t_{1}-D T\right)}{T}$.

There are three cases for interest charged as follows:

\section{Case (i) $t_{M} \leq T$}

See Fig. 2 for the present position of inventory.

Annual interest payable is

$$
\begin{aligned}
& =\frac{c I_{c}}{T}\left[\int_{M}^{t_{1}} I_{1}(t) d t+\int_{t_{1}}^{T} I_{2}(t) d t\right] \\
& =\frac{c I_{c}(P-D)\left(t_{1}^{2}-M^{2}\right)}{2 T}+\frac{c I_{c} D}{T}\left(\frac{T^{2}}{2}-T t_{1}+\frac{t_{1}^{2}}{2}+\frac{T t_{1}^{2}}{2}-\frac{T^{2} t_{1}}{2}+\frac{T^{2} t_{1}^{2}}{4}\right) .
\end{aligned}
$$




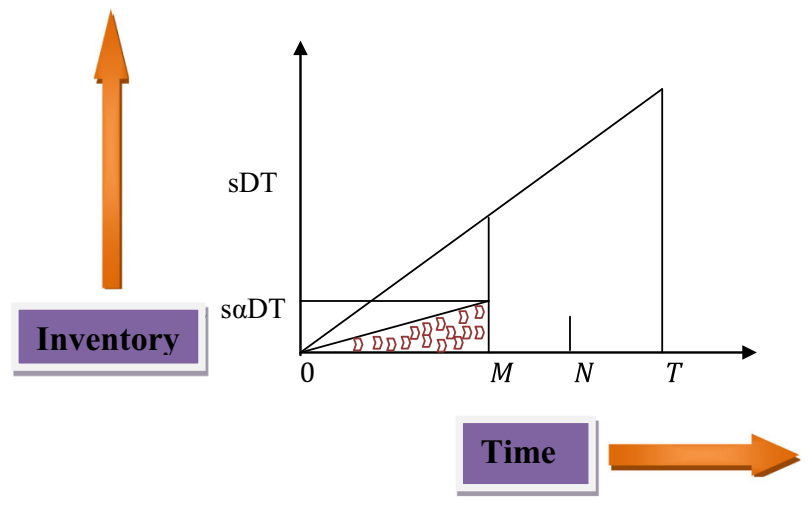

Fig. 7 Total interest earned while $M \leq T$

Case (ii) $M \leq T \leq t_{M}$

See Fig. 3 for the present position of inventory.

Annual interest payable is

$$
\begin{aligned}
& =\frac{c I_{c}}{T}\left[\int_{M}^{T} I_{2}(t) d t\right] \\
& =\frac{c I_{c} D}{T}\left(\frac{T^{2}}{2}-T M+\frac{M^{2}}{2}+\frac{T M^{2}}{2}-\frac{T^{2} M}{2}+\frac{T^{2} M^{2}}{4}\right) .
\end{aligned}
$$

Case (iii) $0<T \leq M$

For this case, the annual interest payable is 0 .

There are three cases for interest earned per year as follows:

Case (a) $t_{M} \leq T$

See Fig. 7 for the present position of inventory.

Annual interest earned $=\frac{s I_{e} D M^{2} \alpha}{2 T}$.

Case (b) $M \leq T \leq t_{M}$

Annual interest earned $=\frac{s I_{e} D M^{2} \alpha}{2 T}$. Which is same as Case (a).

Case (c) $0<T \leq M$

See Fig. 8 for the present position of inventory.

Annual interest earned is

$$
\begin{aligned}
& =\frac{s I_{e}}{T}\left[\frac{D T^{2} \alpha}{2}+\alpha D T(M-T)\right] \\
& =s I_{e} D \alpha\left[M-\frac{T}{2}\right] .
\end{aligned}
$$

Annual total cost of the retailer is given by

$$
\begin{aligned}
& \operatorname{TRC}(T)= \text { Ordering cost }+ \text { Holding cost }+ \text { Deterioration cost }+ \text { Interest payable } \\
& \text {-Interest earned. } \\
& \operatorname{TRC}(T)=\left\{\begin{array}{ll}
T R C_{5}(T) ; & \text { if } T \geq t_{M} \\
T R C_{6}(T) ; & \text { if } M \leq T \leq t_{M} \\
T R C_{7}(T) ; & \text { if } 0<T \leq M
\end{array}\right\}
\end{aligned}
$$


where the costs are given by

$$
\begin{aligned}
T R C_{5}(T)= & \frac{A}{T}+\frac{h(P-D) t_{1}{ }^{2}}{2 T}+\frac{h D}{T}\left(\frac{T^{2}}{2}-T t_{1}+\frac{t_{1}{ }^{2}}{2}+\frac{T t_{1}{ }^{2}}{2}-\frac{T^{2} t_{1}}{2}+\frac{T^{2} t_{1}{ }^{2}}{4}\right) \\
& +\frac{c I_{c}(P-D)\left(t_{1}{ }^{2}-M^{2}\right)}{2 T}+\frac{c I_{c} D}{T}\left(\frac{T^{2}}{2}-T t_{1}+\frac{t_{1}{ }^{2}}{2}+\frac{T t_{1}{ }^{2}}{2}-\frac{T^{2} t_{1}}{2}\right. \\
& \left.+\frac{T^{2} t_{1}{ }^{2}}{4}\right)-\frac{s I_{e} D M^{2} \alpha}{2 T}+\frac{c\left(P t_{1}-D T\right)}{T}, \\
T R C_{6}(T)= & \frac{A}{T}+\frac{h(P-D) t_{1}{ }^{2}}{2 T}+\frac{h D}{T}\left(\frac{T^{2}}{2}-T t_{1}+\frac{t_{1}^{2}}{2}+\frac{T t_{1}^{2}}{2}-\frac{T^{2} t_{1}}{2}+\frac{T^{2} t_{1}{ }^{2}}{4}\right) \\
& +\frac{c I_{c} D}{T}\left(\frac{T^{2}}{2}-T M+\frac{M^{2}}{2}+\frac{T M^{2}}{2}-\frac{T^{2} M}{2}+\frac{T^{2} M^{2}}{4}\right)-\frac{s I_{e} D M^{2} \alpha}{2 T} \\
& +\frac{c\left(P t_{1}-D T\right)}{T},
\end{aligned}
$$

and

$$
\begin{aligned}
T R C_{7}(T)= & \frac{A}{T}+\frac{h(P-D) t_{1}^{2}}{2 T}+\frac{h D}{T}\left(\frac{T^{2}}{2}-T t_{1}+\frac{t_{1}^{2}}{2}+\frac{T t_{1}^{2}}{2}-\frac{T^{2} t_{1}}{2}+\frac{T^{2} t_{1}{ }^{2}}{4}\right) \\
& +\frac{c\left(P t_{1}-D T\right)}{T}-s I_{e} D \alpha\left[M-\frac{T}{2}\right] .
\end{aligned}
$$

For this case, $T R C_{5}\left(t_{M}\right)=T R C_{6}\left(t_{M}\right), T R C_{6}\left(t_{M}\right)=T R C_{7}\left(t_{M}\right)$, and $T R C(T), T R C_{5}(T)$, $T R C_{6}(T)$, and $T R C_{7}(T)$ are well defined for $T>0$.

Lemma For a continuous function $f(t)$ on $(a, b)$ and $\frac{d f(t)}{d t}=0, f(t)$ will be convex.

Proof To prove the above Lemma, there are two cases i.e., $M \geq N$ and $M<N$.

$M \geq N$

For this case, four subcases are appeared which are as follows:

Case (1) $\quad T \geq t_{M}$

Case (2) $\quad M \leq T \leq t_{M}$
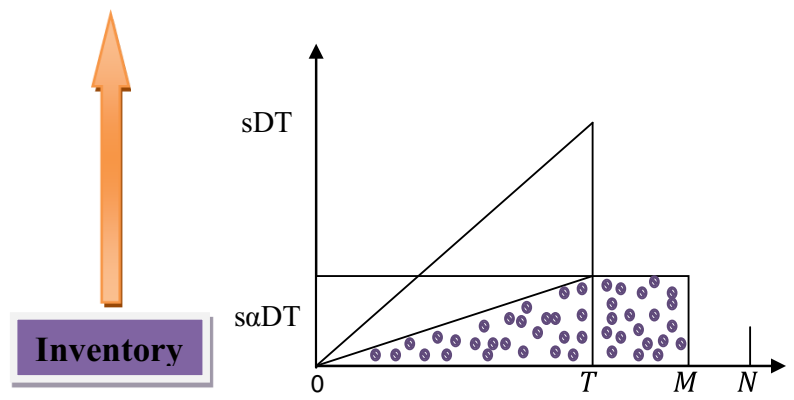

Time

Fig. 8 Total interest earned while $T \leq M$ 


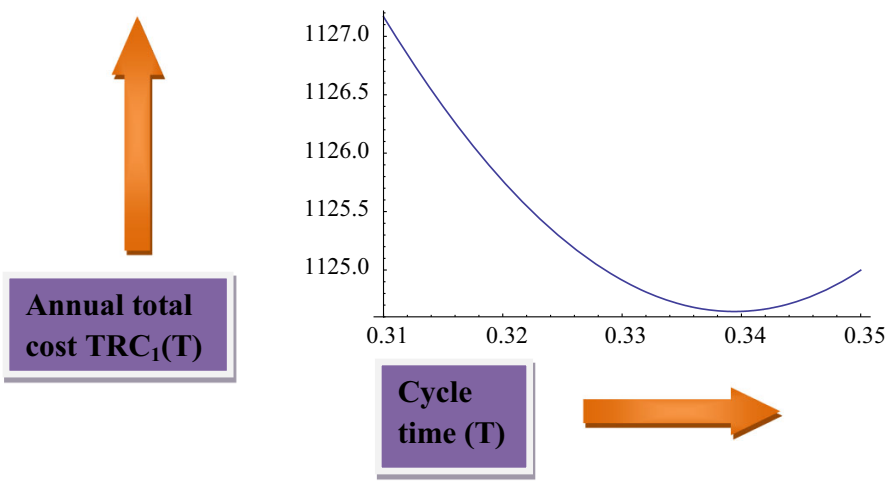

Fig. 9 Annual total cost $T R C_{1}(T)$ versus cycle time (T)

Case (3) $\quad N \leq T \leq M$

Case (4) $\quad 0<T \leq N$

$M<N$

Three subcases are discussed for this case which are given below:

Case (1) $T \geq t_{M}$

Case (2) $M \leq T \leq t_{M}$

Case (3) $0<T \leq M$

The proof of the convexity of all cost functions $T R C_{1}(T), T R C_{2}(T), T R C_{3}(T), T R C_{4}(T)$, $T R C_{5}(T), T R C_{6}(T)$, and $T R C_{7}(T)$ are given in Appendix.

\section{Numerical Examples}

The following numerical examples are provided to illustrate the proposed model. The retailer's annual total cost are determined for seven different cases.

Example 1 Let $A=\$ 200 /$ order, $D=2,500$ units/year, $P=3,000$ units/year, $s=\$ 75 /$ unit, $c=\$ 50 /$ unit, $h=\$ 15 /$ unit/year, $I_{c}=\$ 0.15 / \$ /$ year, $I_{e}=\$ 0.1 /$ /year, $M=0.1$ year, $N=0.05$ year, $\alpha=0.05, \theta=0.05$, then the annual total cost is $T R C_{1}(T)=\$ 2498.8$ and cycle time $T=0.1$ year (Fig. 9).

Example 2 Let $A=\$ 150 /$ order, $D=2,500$ units/year, $P=3,000$ units/year, $s=\$ 75 /$ unit, $c=\$ 50 /$ unit, $h=\$ 15 /$ unit/year, $I_{c}=\$ 0.15 / \$ /$ year, $I_{e}=\$ 0.1 / \$ /$ year, $M=0.1$ year, $N=0.05$ year, $\alpha=0.05, \theta=0.05$, then the annual total cost is $T R C_{2}(T)=\$ 2032.94$ and cycle time $T=0.1$ year (Fig. 10).

Example 3 Let $A=\$ 100 /$ order, $D=2,500$ units/year, $P=4,000$ units/year, $s=\$ 75 /$ unit, $c=\$ 50 /$ unit, $h=\$ 15 /$ unit/year, $I_{c}=\$ 0.15 / \$ /$ year, $I_{e}=\$ 0.1 /$ /year, $M=0.1$ year, $N=0.05$ year, $\alpha=0.05, \theta=0.05$, then the annual total cost is $T R C_{3}(T)=\$ 2481.5$ and cycle time $T=0.05$ year (Fig. 11). 

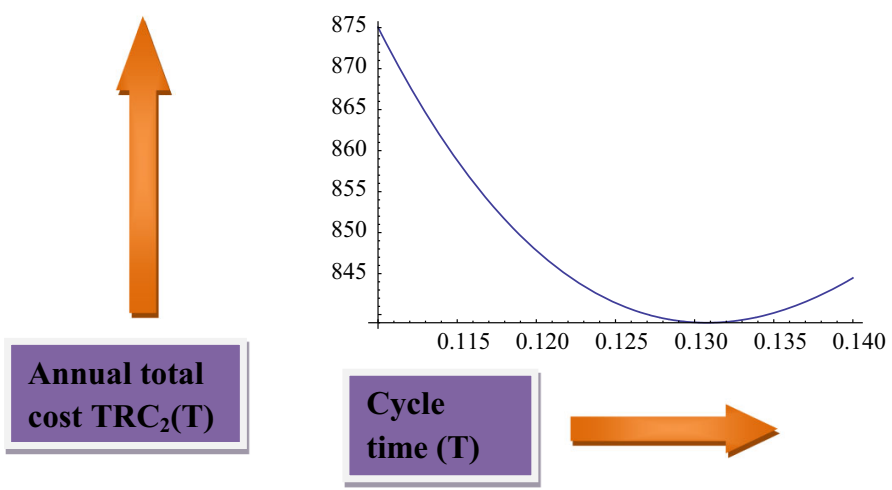

Fig. 10 Annual total cost $T R C_{2}(T)$ versus cycle time $(T)$
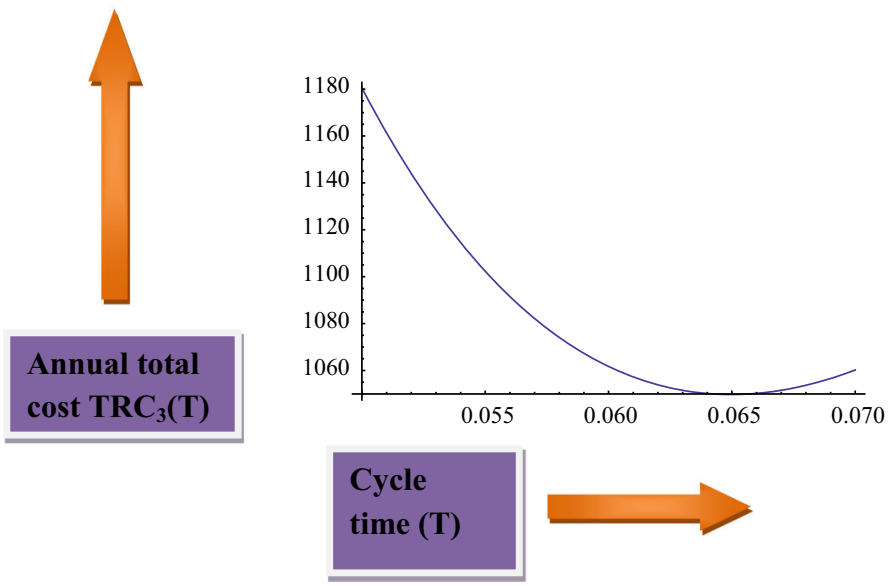

Fig. 11 Annual total cost $T R C_{3}(T)$ versus cycle time $(T)$

Example 4 Let $A=\$ 50 /$ order, $D=2,500$ units/year, $P=4,000$ units/year, $s=\$ 100 /$ unit, $c=\$ 50 /$ unit, $h=\$ 15 /$ unit/year, $I_{c}=\$ 0.15 / \$ /$ year, $I_{e}=\$ 0.12 /$ /year, $M=0.12$ year, $N=0.08$ year, $\alpha=0.05, \theta=0.05$, then the annual total cost is $T R C_{4}(T)=\$ 726.11$ and cycle time $T=0.05$ year (Fig. 12).

Example 5 Let $A=\$ 150 /$ order, $D=2,500$ units/year, $P=3,000$ units/year, $s=\$ 75 /$ unit, $c=\$ 50 /$ unit, $h=\$ 15 /$ unit/year, $I_{c}=\$ 0.15 / \$ /$ year, $I_{e}=\$ 0.1 / \$ /$ year, $M=0.02$ year, $N=0.05$ year, $\alpha=0.05, \theta=0.05$, then the annual total cost is $T R C_{5}(T)=\$ 2886.75$ and cycle time $T=0.1$ year (Fig. 13).

Example 6 Let $A=\$ 100 /$ order, $D=2,500$ units/year, $P=3,500$ units/year, $s=\$ 75 /$ unit, $c=\$ 50 /$ unit, $h=\$ 15 /$ unit/year, $I_{c}=\$ 0.15 /$ /year, $I_{e}=\$ 0.1 /$ /year, $M=0.2$ year, $N=0.05$ year, $\alpha=0.05, \theta=0.05$, then the annual total cost is $T R C_{6}(T)=\$ 3901.37$ and cycle time $T=0.1$ year (Fig. 14). 


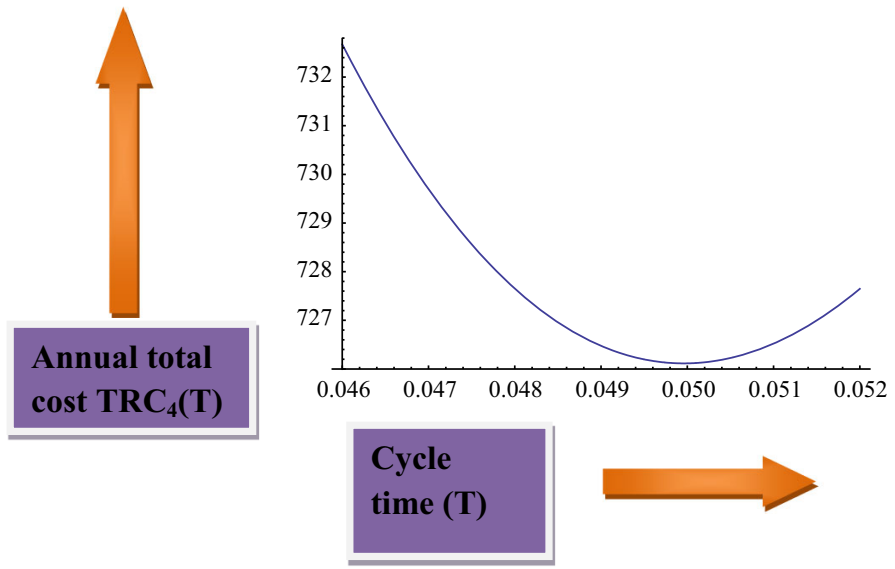

Fig. 12 Annual total cost $T R C_{4}(T)$ versus cycle time ( $T$ )
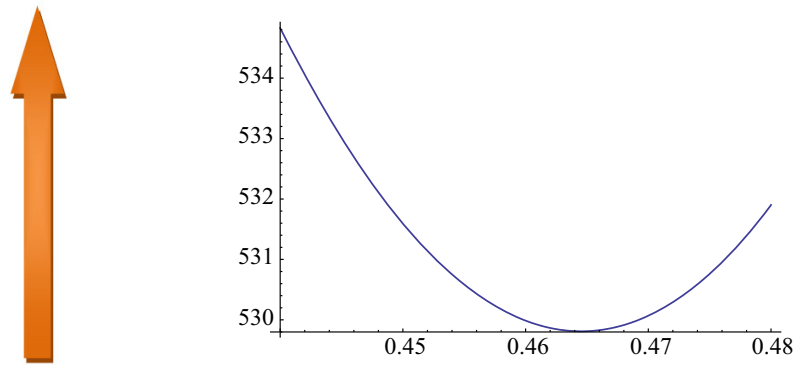

\section{Annual total \\ $\operatorname{cost} \mathrm{TRC}_{5}(\mathrm{~T})$}

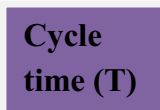

Fig. 13 Annual total cost $T R C_{5}(T)$ versus cycle time $(T)$

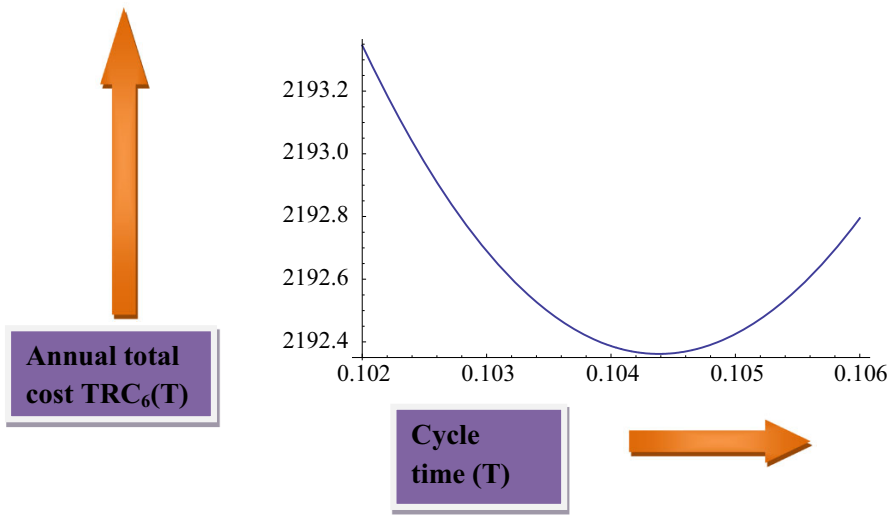

Fig. 14 Annual total cost $T R C_{6}(T)$ versus cycle time ( $T$ ) 

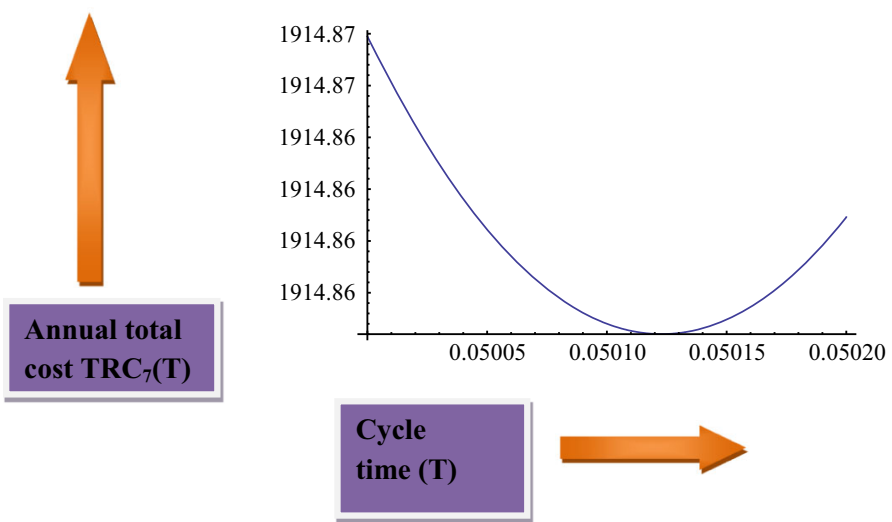

Fig. 15 Annual total cost $T R C_{7}(T)$ versus cycle time $(T)$

Table 2 For the case

$M \leq t_{M} \leq T$

\begin{tabular}{lll}
\hline Parameters & Changes $(\%)$ & TRC $(T)$ \\
\hline \multirow{4}{*}{$A$} & -50 & -80.24 \\
& -25 & - \\
& +25 & 12.18 \\
& +50 & 23.28 \\
& -50 & 8.85 \\
& -25 & 4.53 \\
$s$ & +25 & - \\
& +50 & - \\
& -50 & -150.47 \\
& -25 & -69.05 \\
& +25 & - \\
& +50 & - \\
& -50 & - \\
& -25 & - \\
& +25 & -104.04 \\
& +50 &
\end{tabular}

Example 7 Let $A=\$ 50 /$ order, $D=2,500$ units/year, $P=4,000$ units/year, $s=\$ 100 /$ unit, $c=\$ 50 /$ unit, $h=\$ 15 /$ unit/year, $I_{c}=\$ 0.15 / \$ /$ year, $I_{e}=\$ 0.1 / \$ /$ year, $M=0.1$ year, $N=0.2$ year, $\alpha=0.05, \theta=0.05$, then the annual total cost is $T R C_{7}(T)=\$ 1914.86$ and cycle time $T=0.05$ year (Fig. 15).

\section{Sensitivity Analysis}

In this section, the sensitivity analysis of the key parameters of this proposed model is discussed. See Tables 2, 3, 4, 5, 6, 7, and 8 for sensitivity analysis of key parameters for each seven cases.

- The annual total cost $T R C_{1}(T)$ increases if ordering cost $A$ increases. 
Table 3 For the case $M \leq T \leq t_{M}$

- Refers to infeasible solution

Table 4 For the case $N \leq T \leq M$

- Refers to infeasible solution

\begin{tabular}{lll}
\hline Parameters & Changes $(\%)$ & T R $C_{2}(T)$ \\
\hline \multirow{4}{*}{$A$} & -50 & -15287.6 \\
& -25 & -36.51 \\
& +25 & 32.30 \\
& +50 & - \\
& -50 & 38.83 \\
$s$ & -25 & 20.05 \\
& +25 & - \\
& +50 & - \\
& -50 & -106.242 \\
& -25 & - \\
& +25 & - \\
& +50 & 66.82 \\
& -50 & 46.47 \\
& -25 & - \\
& +25 & - \\
& +50 & - \\
\hline & &
\end{tabular}

\begin{tabular}{lll}
\hline Parameters & Changes $(\%)$ & TRC $C_{3}(T)$ \\
\hline \multirow{4}{*}{$A$} & -50 & -82.81 \\
& -25 & -38.74 \\
& +25 & 34.95 \\
& +50 & - \\
& -50 & 84.53 \\
& -25 & 42.37 \\
$s$ & +25 & -42.53 \\
& +50 & -85.20 \\
& -50 & - \\
& -25 & - \\
& +25 & 33.40 \\
& +50 & 64.34 \\
& -50 & 7.97 \\
& -25 & 4.01 \\
& +25 & -4.06 \\
& +50 & -8.19 \\
& &
\end{tabular}

- As selling price $s$ increases, the annual total cost $T R C_{1}(T)$ decreases gradually. But, for $25 \%$ and $50 \%$ increase of the selling price, the model does not allow feasible results.

- If the purchasing cost $c$ increases, then the annual total cost $T R C_{1}(T)$ increases. But, if we increase the purchasing cost for $25 \%$ more, we obtain non-feasible result. Therefore, there is a boundary to increase the purchasing cost of products.

- An increasing value in the holding cost $h$ decreases the annual total cost $T R C_{1}(T)$.

- The annual total cost $T R C_{2}(T)$ increases as soon as the ordering cost $A$ increases. 
Table 5 For the case $0<T \leq N$

\begin{tabular}{lll}
\hline Parameters & Changes $(\%)$ & $T R C_{4}(T)$ \\
\hline \multirow{4}{*}{$A$} & -50 & -81.27 \\
& -25 & -37.04 \\
& +25 & 32.45 \\
& +50 & 61.64 \\
& -50 & 88.30 \\
& -25 & 44.15 \\
$s$ & +25 & -44.16 \\
& +50 & -88.33 \\
& -50 & - \\
& -25 & -42.28 \\
& +25 & 36.70 \\
& +50 & 69.58 \\
& -50 & 8.61 \\
& -25 & 4.35 \\
& +25 & -4.43 \\
& +50 & -8.95 \\
\hline & &
\end{tabular}

Table 6 For the case $T \geq t_{M}$

\begin{tabular}{lrr}
\hline Parameters & Changes $(\%)$ & $T R C_{5}(T)$ \\
\hline \multirow{4}{*}{$A$} & -50 & -31.23 \\
& -25 & -15.41 \\
& +25 & 15.08 \\
& +50 & 29.88 \\
& -50 & 0.038 \\
& -25 & 0.019 \\
$s$ & +25 & -0.019 \\
& +50 & -0.038 \\
& -50 & -286.83 \\
& -25 & -137.58 \\
& +25 & 123.66 \\
& +50 & 225.61 \\
& -50 & 202.19 \\
& -25 & 114.70 \\
& +25 & -125.25 \\
& +50 & -255.45 \\
\hline
\end{tabular}

- As selling price $s$ increases, the annual total cost $T R C_{2}(T)$ decreases gradually.

- If the purchasing cost $c$ increases, then the annual total cost $T R C_{2}(T)$ increases.

- If the ordering cost $A$ increases, the annual total cost $T R C_{3}(T)$ increases.

- For this case, the annual total cost $T R C_{3}(T)$ decreases if the selling price $s$ increases. The selling price is more sensitive than other parameters here.

- If the purchasing cost $c$ increases, then the annual total cost $T R C_{3}(T)$ increases.

- As holding cost $h$ decreases, the annual total cost $T R C_{3}(T)$ increases. The positive change is grater than the negative change for the holding cost $h$. 
Table 7 For the case

$M \leq T \leq t_{M}$

- Refers to infeasible solution

Table 8 For the case

$0<T \leq M$

- Refers to infeasible solution

\begin{tabular}{lll}
\hline Parameters & Changes $(\%)$ & TRC $6(T)$ \\
\hline & -50 & -23.47 \\
& -25 & - \\
$A$ & +25 & 10.59 \\
& +50 & - \\
& -50 & 1.23 \\
& -25 & 0.62 \\
$s$ & +25 & -0.62 \\
& +50 & -1.25 \\
& -50 & - \\
& -25 & - \\
$c$ & +25 & - \\
& +50 & -37.71 \\
& -50 & 10.03 \\
& -25 & 5.09 \\
$h$ & +25 & -5.27 \\
& +50 & -10.75 \\
\hline
\end{tabular}

\begin{tabular}{lll}
\hline Parameters & Changes $(\%)$ & $T R C_{7}(T)$ \\
\hline \multirow{4}{*}{$A$} & -50 & -30.72 \\
& -25 & -14.00 \\
& +25 & 12.26 \\
& +50 & 23.30 \\
& -50 & -2.44 \\
$s$ & -25 & 1.22 \\
& +25 & -1.22 \\
& +50 & -2.45 \\
& -50 & - \\
& -25 & -16.10 \\
& +25 & 13.95 \\
& +50 & 26.45 \\
& -50 & 3.28 \\
& -25 & 1.65 \\
& +25 & -1.69 \\
& +50 & -3.41
\end{tabular}

- The negative change for the parameter ordering cost $A$ is grater than the positive change. The annual total cost $T R C_{4}(T)$ increases if ordering cost $A$ increases.

- If selling price $s$ increases, the annual total cost $T R C_{4}(T)$ decreases. The percentage changes in positive change and negative change for selling price $s$ are almost similar.

- The annual total cost $T R C_{4}(T)$ increases if the purchasing cost $c$ increases.

- The positive change in the holding $\cos t h$ is much more greater than the negative change. An increasing value in the holding cost $h$ decreases the annual total cost $T R C_{4}(T)$. 
- The negative change for the parameter ordering cost $A$ is grater than the positive change. The annual total cost $T R C_{5}(T)$ increases if ordering cost $A$ increases.

- The percentage change in the parameter $s$ is same for both the positive and negative change. If the selling price increases then the annual total cost $T R C_{5}(T)$ decreases gradually.

- This model is more sensitive for the negative change than the positive change for the parameter $c$. If the purchasing cost $c$ increases, then the annual total cost $T R C_{5}(T)$ increases.

- The positive change is much more than the negative change for the parameter $h$. An increasing value in the holding cost $h$ decreases the annual total cost $T R C_{5}(T)$.

- As the ordering cost $A$ increases, the annual total cost $T R C_{6}(T)$ increases.

- The negative change is less than the positive change for selling price $s$. If the selling price $s$ increases, the annual total cost $T R C_{6}(T)$ decreases.

- The positive change is bigger than the negative change for holding cost $h$. An increasing value in the holding cost $h$ decreases the annual total cost $T R C_{6}(T)$.

- The negative change is greater than the positive change for ordering cost $A$. The annual total cost $T R C_{7}(T)$ increases if ordering cost $A$ increases.

- As selling price increases, the annual total $\operatorname{cost} T R C_{7}(T)$ decreases gradually. The positive change is greater than the negative change for selling price $s$.

- If the purchasing cost $c$ increases, then the annual total cost $T R C_{7}(T)$ increases.

- This model is more sensitive for positive change than the negative change in holding cost $h$. An increasing value in the holding cost $h$ decreases the annual total cost $T R C_{7}(T)$.

\section{Conclusions}

This study was developed based on the strategy that supplier offered their retailer full tradecredit policy, but the retailer offered their customer partial trade-credit policy. Because of allowing partial trade-credit policy, retailer might delay the payment upto the last moment of permissible period offered by supplier. Deterioration of products was taken as exponential rather than the constant over whole time. This study minimized the retailer's annual total cost for finite replenishment rate. For each different cases, the model is optimized with the help of analytical procedure and finally, several numerical results are given to illustrate the retailer's cost function. This model can be extended in many different ways by considering shortages and probabilistic demand. It will be a fruitful research if we can add preservation cost to reduce the deterioration of products.

Acknowledgments The authors wish to thank the reviewers for their very helpful comments and suggestions to improve the paper. The research of second author was supported by Rajiv Gandhi National Fellowship (F117.1/2012-13/RGNF-2012-13-ST-WES-15873). This work was supported by the research fund of Hanyang University (HY-2014-N, Project number 201400000002202) for new Faculty members.

\section{Appendix}

$M \geq N$

Case (1) $M \leq t_{1}$ i.e., $M \leq t_{M} \leq T$ Here

$$
\frac{d T R C_{1}(T)}{d T}=\frac{f_{1}(T)}{T^{2}}
$$


where

$$
\begin{aligned}
f_{1}(T)= & -A-\frac{h(P-D) t_{1}{ }^{2}}{2}+\left(h+c I_{c}\right) D T^{2}\left(\frac{1}{2}-\frac{3 T^{2}}{4}-\frac{t_{1}{ }^{2}}{2 T^{2}}-\frac{t_{1}}{2}+\frac{t_{1}{ }^{2}}{4}\right) \\
& -\frac{c I_{c}(P-D)\left(t_{1}{ }^{2}-M^{2}\right)}{2}-c P t_{1}+\frac{s I_{e} D}{2}\left[M^{2}-(1-\alpha) N^{2}\right]
\end{aligned}
$$

To determine the optimal value of $T$ say $T_{1}^{*}$, solving the equation $f_{1}(T)=0$.

From the equation of $f_{1}(T)$

$$
\frac{d f_{1}(T)}{d T}>0, \text { if } T>0 .
$$

$f_{1}(T)$ is an increasing function on $[0, \infty)$, then $\frac{d T R C_{1}(T)}{d T}$ is an increasing function on $[0, \infty)$. Using Lemma, $T R C_{1}(T)$ is a convex function on $[0, \infty)$.

In addition as $\lim T \rightarrow \infty$, then $f_{1}(T) \rightarrow \infty$. From equation of $f_{1}(T)$

$$
f_{1}(0)=-A-\frac{h(P-D) t_{1}^{2}}{2}+\frac{s I_{e} D}{2}\left[M^{2}-(1-\alpha) N^{2}\right]-\frac{c I_{c}(P-D)\left(t_{1}^{2}-M^{2}\right)}{2}-c P t_{1}
$$

Then

$$
\begin{aligned}
\frac{d T R C_{1}(T)}{d T} & <0 ; \quad \text { if } T \in\left[0, T_{1}^{*}\right), \\
& =0 ; \quad \text { if } T=T_{1}^{*} \\
& >0 ; \quad \text { if } T \in\left(T_{1}^{*}, \infty\right) .
\end{aligned}
$$

By applying the intermediate value theorem, an optimal solution $T_{1}^{*}$ exists and is unique. Case (2) $M \leq T \leq t_{M}$

$$
\frac{d T R C_{2}(T)}{d T}=\frac{f_{2}(T)}{T^{2}}
$$

where

$$
\begin{aligned}
f_{2}(T)= & -A-\frac{h(P-D) t_{1}{ }^{2}}{2}+\left(h+c I_{c}\right) D T^{2}\left(\frac{1}{2}-\frac{3 T^{2}}{4}\right)+\left(h+c I_{c}\right) D T^{2}\left(\frac{\left(t_{1}{ }^{2}+M^{2}\right)}{4}\right. \\
& \left.-\frac{\left(t_{1}{ }^{2}+M^{2}\right)}{2 T^{2}}-\frac{\left(t_{1}+M\right)}{2}\right)-c P t_{1}+\frac{s I_{e} D}{2}\left[M^{2}-(1-\alpha) N^{2}\right]
\end{aligned}
$$

To determine the optimal value of $T$ say $T_{2}^{*}$, solving the equation $f_{2}(T)=0$. From the equation of $f_{2}(T)$

$$
\frac{d f_{2}(T)}{d T}>0, \quad \text { if } \quad T>0
$$

$f_{2}(T)$ is an increasing function on $[0, \infty)$, then $\frac{d T R C_{2}(T)}{d T}$ is an increasing function on $[0, \infty)$. Using Lemma, $T R C_{2}(T)$ is a convex function on $[0, \infty)$.

In addition as $\lim T \rightarrow \infty$, then $f_{2}(T) \rightarrow \infty$.

$$
f_{2}(0)=-A-\frac{h(P-D) t_{1}^{2}}{2}-c P t_{1}+\frac{s I_{e} D}{2}\left[M^{2}-(1-\alpha) N^{2}\right] .
$$

Then

$$
\frac{d T R C_{2}(T)}{d T}<0 ; \quad \text { if } T \in\left[0, T_{2}^{*}\right)
$$




$$
\begin{array}{ll}
=0 ; & \text { if } T=T_{2}^{*}, \\
>0 ; & \text { if } T \in\left(T_{2}^{*}, \infty\right) .
\end{array}
$$

Using intermediate value theorem, an optimal solution $T_{2}^{*}$ exists and is unique.

Case (3) $N \leq T \leq M$

$$
\frac{d T R C_{3}(T)}{d T}=\frac{f_{3}(T)}{T^{2}}
$$

where

$$
\begin{aligned}
f_{3}(T)= & -A-\frac{h(P-D) t_{1}^{2}}{2}+h D T^{2}\left(\frac{1}{2}-\frac{3 T^{2}}{4}-\frac{t_{1}}{2}-\frac{t_{1}^{2}}{2 T^{2}}+\frac{t_{1}{ }^{2}}{4}\right)-c P t_{1} \\
& +\frac{s I_{e} D}{2}\left(M^{2}-(1-\alpha) N^{2}\right)
\end{aligned}
$$

To determine the optimal value of $T$ say $T_{3}^{*}$, solving the equation $f_{3}(T)=0$. From the equation of $f_{3}(T)$

$$
\frac{d f_{3}(T)}{d T}>0, \text { if } T>0 .
$$

$f_{3}(T)$ is an increasing function on $[0, \infty)$, then $\frac{d T R C_{3}(T)}{d T}$ is an increasing function on $[0, \infty)$. Using Lemma, $T R C_{3}(T)$ is a convex function on $[0, \infty)$.

In addition as $\lim T \rightarrow \infty$, then $f_{3}(T) \rightarrow \infty$.

$$
f_{3}(0)=-A-\frac{h(P-D) t_{1}^{2}}{2}-c P t_{1}+\frac{s I_{e} D}{2}\left(M^{2}-(1-\alpha) N^{2}\right) \text {. }
$$

Then

$$
\begin{aligned}
\frac{d T R C_{3}(T)}{d T}<0 ; & \text { if } \quad T \in\left[0, T_{3}^{*}\right), \\
=0 ; & \text { if } \quad T=T_{3}^{*}, \\
>0 ; & \text { if } \quad T \in\left(T_{3}^{*}, \infty\right) .
\end{aligned}
$$

The intermediate value theorem states that the optimal solution $T_{3}^{*}$ exists and is unique.

Case (4) $0<T \leq N$

$$
\frac{d T R C_{4}(T)}{d T}=\frac{f_{4}(T)}{T^{2}}
$$

where

$$
\begin{aligned}
f_{4}(T)= & -A-\frac{h(P-D) t_{1}^{2}}{2}+h D T^{2}\left(\frac{1}{2}-\frac{3 T^{2}}{4}-\frac{t_{1}}{2}-\frac{t_{1}{ }^{2}}{2 T^{2}}+\frac{t_{1}{ }^{2}}{4}\right)-c P t_{1} \\
& +\frac{s I_{e} D \alpha T^{2}}{2}
\end{aligned}
$$

To determine the optimal value of $T$ say $T_{4}^{*}$, solving the equation $f_{4}(T)=0$. From the equation of $f_{4}(T)$

$$
\frac{d f_{4}(T)}{d T}>0, \quad \text { if } T>0
$$

$f_{4}(T)$ is an increasing function on $[0, \infty)$, then $\frac{d T R C_{4}(T)}{d T}$ is an increasing function on $[0, \infty)$. Using Lemma $1, T R C_{4}(T)$ is a convex function on $[0, \infty)$. 
In addition as $\lim T \rightarrow \infty$, then $f_{4}(T) \rightarrow \infty$. From equation of $f_{4}(T)$

$$
f_{4}(0)=-A-\frac{h(P-D) t_{1}^{2}}{2}-c P t_{1} .
$$

Then

$$
\begin{aligned}
\frac{d T R C_{4}(T)}{d T} & <0 ; \quad \text { if } T \in\left[0, T_{4}^{*}\right), \\
& =0 ; \quad \text { if } T=T_{4}^{*}, \\
& >0 ; \quad \text { if } T \in\left(T_{4}^{*}, \infty\right) .
\end{aligned}
$$

The intermediate value theorem states that the optimal solution $T_{4}^{*}$ exists and is unique.

$M<N$

Case (1) $T \geq t_{M}$

$$
\frac{d T R C_{5}(T)}{d T}=\frac{f_{5}(T)}{T^{2}}
$$

where

$$
\begin{aligned}
f_{5}(T)= & -A-\frac{h(P-D) t_{1}^{2}}{2}+\left(h+c I_{c}\right) D T^{2}\left(\frac{1}{2}-\frac{3 T^{2}}{4}-\frac{t_{1}{ }^{2}}{2 T^{2}}-\frac{t_{1}}{2}+\frac{t_{1}{ }^{2}}{4}\right) \\
& -\frac{c I_{c}(P-D)\left(t_{1}{ }^{2}-M^{2}\right)}{2}-c P t_{1}+\frac{s I_{e} D \alpha M^{2}}{2}
\end{aligned}
$$

To determine the optimal value of $T$ say $T_{5}^{*}$, solving the equation $f_{5}(T)=0$.

From the equation of $f_{5}(T), \frac{d f_{5}(T)}{d T}>0$ if $T>0 . f_{5}(T)$ is an increasing function on $[0, \infty)$, then $\frac{d T R C_{5}(T)}{d T}$ is an increasing function on $[0, \infty)$. Using Lemma, $T R C_{5}(T)$ is a convex function on $[0, \infty)$.

Thus, $\lim T \rightarrow \infty$, then $f_{5}(T) \rightarrow \infty$.

From equation of $f_{5}(T)$,

$$
f_{5}(0)=-A-\frac{h(P-D) t_{1}^{2}}{2}-\frac{c I_{c}(P-D)\left(t_{1}^{2}-M^{2}\right)}{2}-c P t_{1}+\frac{s I_{e} D \alpha M^{2}}{2} .
$$

Then

$$
\begin{aligned}
\frac{d T R C_{5}(T)}{d T} & <0 ; \quad \text { if } T \in\left[0, T_{5}^{*}\right), \\
& =0 ; \quad \text { if } T=T_{5}^{*}, \\
& >0 ; \quad \text { if } T \in\left(T_{5}^{*}, \infty\right) .
\end{aligned}
$$

Using the intermediate value theorem, there exist a unique optimal solution $T_{5}^{*}$.

Case (2) $M \leq T \leq t_{M}$

$$
\frac{d T R C_{6}(T)}{d T}=\frac{f_{6}(T)}{T^{2}}
$$

where

$$
\begin{aligned}
f_{6}(T)= & -A-\frac{h(P-D) t_{1}{ }^{2}}{2}+\left(h+c I_{c}\right) D T^{2}\left(\frac{1}{2}-\frac{3 T^{2}}{4}\right)+\left(h+c I_{c}\right) D T^{2}\left(\frac{\left(t_{1}{ }^{2}+M^{2}\right)}{4}\right. \\
& \left.-\frac{\left(t_{1}{ }^{2}+M^{2}\right)}{2 T^{2}}-\frac{\left(t_{1}+M\right)}{2}\right)-c P t_{1}+\frac{s I_{e} D \alpha M^{2}}{2}
\end{aligned}
$$


To determine the optimal value of $T$ say $T_{6}^{*}$, solving the equation $f_{6}(T)=0$.

From the equation of $f_{6}(T), \frac{d f_{6}(T)}{d T}>0$ if $T>0$.

$f_{6}(T)$ is an increasing function on $[0, \infty)$, then $\frac{d T R C_{6}(T)}{d T}$ is an increasing function on $[0, \infty)$. Using Lemma, $T R C_{6}(T)$ is a convex function on $[0, \infty)$.

Thus, $f_{6}(T) \rightarrow \infty$ as $\lim T \rightarrow \infty$.

$$
f_{6}(0)=-A-\frac{h(P-D) t_{1}^{2}}{2}-c P t_{1}+\frac{s I_{e} D \alpha M^{2}}{2} \text {. }
$$

Then

$$
\begin{aligned}
\frac{d T R C_{6}(T)}{d T} & <0 ; \quad \text { if } T \in\left[0, T_{6}^{*}\right), \\
& =0 ; \quad \text { if } T=T_{6}^{*}, \\
& >0 ; \quad \text { if } T \in\left(T_{6}^{*}, \infty\right) .
\end{aligned}
$$

From the intermediate value theorem, a unique optimal solution $T_{6}^{*}$ exists.

Case (3) $0<T \leq M$

$$
\frac{d T R C_{7}(T)}{d T}=\frac{f_{7}(T)}{T^{2}}
$$

where

$$
\begin{aligned}
f_{7}(T)= & -A-\frac{h(P-D) t_{1}{ }^{2}}{2}+h D T^{2}\left(\frac{1}{2}-\frac{3 T^{2}}{4}-\frac{t_{1}{ }^{2}}{2 T^{2}}-\frac{t_{1}}{2}+\frac{t_{1}{ }^{2}}{4}\right)-c P t_{1} \\
& +\frac{s I_{e} D \alpha}{2}
\end{aligned}
$$

To determine the optimal value of $T$ say $T_{7}^{*}$, solving the equation $f_{7}(T)=0$.

From the equation of $f_{7}(T), \frac{d f_{7}(T)}{d T}>0$, if $T>0$.

As $f_{7}(T)$ is an increasing function on $[0, \infty)$, then $\frac{d T R C_{7}(T)}{d T}$ is an increasing function on $[0, \infty)$. Using the Lemma, $\operatorname{TR} C_{7}(T)$ is a convex function on $[0, \infty)$.

Therefore $f_{7}(T) \rightarrow \infty$ as $\lim T \rightarrow \infty$. Now

$$
f_{7}(0)=-A-\frac{h(P-D) t_{1}^{2}}{2}-c P t_{1}+\frac{s I_{e} D \alpha}{2} .
$$

Then

$$
\begin{aligned}
\frac{d T R C_{7}(T)}{d T} & <0 ; \text { if } T \in\left[0, T_{7}^{*}\right), \\
& =0 ; \text { if } T=T_{7}^{*}, \\
& >0 ; \text { if } T \in\left(T_{7}^{*}, \infty\right) .
\end{aligned}
$$

Utilizing the intermediate value theorem, a unique optimal solution $T_{7}^{*}$ is obtained.

\section{References}

1. Arcelus, F.J., Shah, N.H., Srinivasan, G.: Retailer's pricing, credit and inventory policies for deteriorating items in response to temporary price/credit incentives. Int. J. Prod. Econ. 81-82, 153-162 (2003)

2. Balkhi, Z.: Optimal economic ordering policy with deteriorating items under different supplier tradecredits for finite horizon case. Int. J. Prod. Econ. 133, 216-223 (2011) 
3. Chang, C.T., Ouyang, L.Y., Teng, J.T.: An EOQ model for deteriorating items under supplier credits linked to ordering quantity. Appl. Math. Model. 27, 983-996 (2003)

4. Chen, S.H., Cárdenas-Barrón, L.E., Teng, J.T.: Retailer's economic order quantity when the supplier offers conditionally permissible delay-in-payments link to order quantity. Int. J. Prod. Econ. 155, 284291 (2014)

5. Chung, K.J.: Comments on the EOQ model under retailer partial trade-credit policy in the supply chain. Int. J. Prod. Econ. 114, 308-312 (2008)

6. Mahata, G.C.: An EPQ-based inventory model for exponentially deteriorating items under retailer partial trade-credit policy in supply chain. Expert Syst. Appl. 39, 3537-3550 (2012)

7. Ouyang, L.Y., Wu, K.S., Yang, C.T.: A study on an inventory model for non-instantaneous deteriorating items with permissible delay in payments. Comput. Ind. Eng. 51, 637-651 (2006)

8. Ouyang, L.Y., Yang, C.T., Chan, Y.L., Cárdenas-Barrón, L.E.: A comprehensive extension of the optimal replenishment decisions under two-levels of trade-credit policy depending on the order quantity. Appl. Math. Comput. 224, 268-277 (2013)

9. Sarkar, B., Gupta, H., Chaudhuri, K., Goyal, S.K.: An integrated inventory model with variable lead time, defective units and delay-in-payments. Appl. Math. Comp. 237, 650-658 (2014)

10. Shah, N.H., Patel, D.G., Shah, D.B.: Optimal pricing and ordering policies for inventory system with two-level trade-credits under price-sensitive trended demand. Int. J. Appl. Comput. Math. (2014). doi:10. 1007/s40819-014-0003-9

11. Soni, H.N., Patel, K.A.: Optimal strategy for an integrated inventory system involving variable production and defective items under retailer partial trade-credit policy. Dec. Sup. Sys. 54, 235-247 (2012)

12. Taft, E.W.: The most economical production lot. Iron Age 101, 1410-1412 (1918)

13. Chang, H.C.: An economic production quantity model with consolidating shipments of imperfect quality items: a note. Int. J. Prod. Econ. 144, 507-509 (2013)

14. Cheng, T.C.E.: An economic production quantity model with flexibility and reliability considerations. Eur. J. Oper. Res. 39, 174-179 (1989)

15. Chiu, S.W., Lin, H.D., Wu, M.F., Yang, J.C.: Determining replenishment lot size and shipment policy for an extended EPQ model with delivery and quality assurance issues. Sci. Iran. 18, 1537-1544 (2011)

16. Goyal, S.K., Cárdenas-Barrón, L.E.: note on: economic production quantity model for items with imperfect quality: a practical approach. Int. J. Prod. Econ. 77, 85-87 (2002)

17. Hall, R.W.: On the integration of production and distribution: economic order and production quantity implications. Trans. Res. Method 30, 387-403 (1996)

18. Pal, B., Sana, S.S., Chaudhuri, K.S.: A mathematical model on EPQ for stochastic demand in an imperfect production system. J. Manuf. Syst. 32, 260-270 (2013)

19. Szendrovits, A.Z., Goyal, S.K.: A unified EPQ model for packaging two items jointly manufactured through several stages. Eur. J. Oper. Res. 7, 249-256 (1981)

20. Cárdenas-Barrón, L.E.: The economic production quantity (EPQ) with shortage derived algebraically. Int. J. Prod. Econ. 70, 289-292 (2001)

21. Teng, J.T., Ouyang, L.Y., Chang, C.T.: Deterministic economic production quantity models with timevarying demand and cost. Appl. Math. Model. 29, 987-1003 (2005)

22. Wee, H.M., Wang, W.T., Lee, M.C., Cárdenas-Barrón, L.E.: Solving a finite horizon EPQ problem with backorders. Appl. Math. Model. 37, 7876-7882 (2013)

23. Chakrabarty, T., Giri, B.C., Chaudhuri, K.S.: an EOQ model for items with Weibull distribution deterioration, shortages and trended demand: an extension of Philip's model. Comput. Oper. Res. 25, 649-657 (1998)

24. Chu, P., Chen, P.S.: A note on inventory replenishment policies for deteriorating items in an exponentially declining market. Comput. Oper. Res. 29, 1827-1842 (2002)

25. Chung, C.J., Wee, H.M.: Short life-cycle deteriorating product remanufacturing in a green supply chain inventory control system. Int. J. Prod. Econ. 129, 195 (2011)

26. Chung, K.J., Cárdenas-Barrón, L.E., Ting, P.S.: An inventory model with non-instantaneous receipt and exponentially deteriorating items for an integrated three layer supply chain system under two-levels of trade-credit. Int. J. Prod. Econ. 155, 310-317 (2014)

27. Chung, K.J., Cárdenas-Barrón, L.E.: The simplified solution procedure for deteriorating items under stock-dependent demand and two-level trade-credit in the supply chain management. Appl. Math. Model. 37, 4653-4660 (2013)

28. Covert, R.P., Philip, G.C.: An EOQ model for items with Weibull distribution deterioration. AIIE Trans. 5, 323-326 (1973)

29. Goyal, S.K., Giri, B.C.: The production-inventory problem of a product with time varying demand, production and deterioration rates. Eur. J. Oper. Res. 147, 549-557 (2003) 
30. Hariga, M.: Optimal EOQ models for deteriorating items with time-varying demand. J. Oper. Res. Soc. 47, 1228-1246 (1996)

31. Mukhopadhyay, S., Mukherjee, R.N., Chaudhuri, K.S., Goyal, S.K., Giri, B.C.: Joint pricing and ordering policy for a deteriorating inventory. Comput. Ind. Eng. 47, 339-349 (2004)

32. Sana, S.S.: Optimal selling price and lot size with time varying deterioration and partial backlogging. Appl. Math. Comput. 217, 185-194 (2010)

33. Sarkar, B.: A production-inventory model with probabilistic deterioration in two-echelon supply chain management. Appl. Math. Model. 37, 3138-3151 (2013)

34. Sarkar, B.: An EOQ model with delay-in-payments and time varying deterioration rate. Math. Comput. Model. 55, 367-377 (2012)

35. Sarkar, B., Saren, S., Wee, H.M.: An inventory model with variable demand, component cost and selling price for deteriorating items. Econ. Model. 30, 306-310 (2013)

36. Sarkar, B., Sarkar, S.: Variable deterioration and demand: an inventory model. Econ. Model. 31, 548-556 (2013)

37. Sarkar, B., Sarkar, S.: An improved inventory model with partial backlogging, time varying deterioration and stock-dependent demand. Econ. Model. 30, 924-932 (2013)

38. Sarkar, M., Sarkar, B.: An economic manufacturing quantity model with probabilistic deterioration in a production system. Econ. Model. 31, 245-252 (2013)

39. Sett, B.K., Sarkar, B., Goswami, A.: A two-warehouse inventory model with increasing demand and time varying deterioration. Sci. Iran. 19, 1969-1977 (2012)

40. Skouri, K., Konstantaras, I., Papachristos, S., Ganas, I.: Inventory models with ramp type demand rate, partial backlogging and Weibull deterioration rate. Eur. J. Oper. Res. 192, 79-92 (2009)

41. Wu, J., Ouyang, L.Y., Cárdenas-Barrón, L.E., Goyal, S.K.: Optimal credit period and lot size for deteriorating items with expiration dates under two-level trade-credit financing. Eur. J. Oper. Res. 237, 898-908 (2014)

42. Sarkar, B., Saren, S., Cárdenas-Barrón, L.E.: An inventory model with trade-credit policy and variable deterioration for fixed lifetime products. Ann. Oper. Res. (2014). doi:10.1007/s10479-014-1745-9

43. De, S.K., Sana, S.S.: Alternative Fuzzy EOQ model with backlogging for selling price and promotional effort sensitive demand. Int. J. App. Comp. Math. (2014). doi:10.1007/s40819-014-0010-x 